\title{
Improved response time of laser etched polymer optical fiber Bragg grating humidity sensor
}

\author{
Wei Zhang ${ }^{\mathrm{a}}$, Xianfeng Chen ${ }^{\mathrm{b}}$, Chen Liu ${ }^{\mathrm{b}}$, Yuanfu Lu ${ }^{\mathrm{b}, \mathrm{c}}$, Marcos Cardoso ${ }^{\mathrm{b}, \mathrm{d}}$, David J. Webb \\ a Aston Institute of Photonic Technology, Aston University, Birmingham, B4 7ET, UK; \\ ${ }^{\mathrm{b}}$ School of Electronic Engineering, Bangor University, Bangor, LL57 1UT, UK; \\ c Shenzhen Institutes of Advanced Technology, Chinese Academy of Sciences, China \\ ${ }^{\mathrm{d}}$ Instituto de Física de São Carlos, University of São Paulo, São Carlos, Brazil
}

\begin{abstract}
The humidity sensor made of polymer optical fiber Bragg grating (POFBG) responds to the water content change in fiber induced by the change of environmental condition. The response time strongly depends on fiber size as the water change is a diffusion process. The ultrashort laser pulses have been providing an effective microfabrication method to achieve spatial localized modification in materials. In this work we used the excimer laser to create different microstructures (slot, D-shape) in POFBG to improve its performance. A significant improvement in the response time has been achieved in a laser etched D-shaped POFBG humidity sensor.
\end{abstract}

Keywords: Fiber Bragg grating, Polymer optical fiber, Laser etching, Response time, Humidity sensing

\section{INTRODUCTION}

The physical and chemical properties of polymeric materials are rather different to silica, potentially making it attractive for researchers to exploit in device and sensing applications. Polymer optical fibers (POFs) provide additional advantages for sensing applications, including high strain limits, high fracture toughness, high flexibility in bending, large negative thermo-optic coefficients and for some materials an affinity for water. Polymers have excellent compatibility with organic materials, giving them great potential for biomedical applications [1]. More recently, fiber Bragg gratings (FBGs) have been inscribed into step index [2] and microstructured POF [3] based on PMMA. Polymer optical fiber Bragg gratings (POFBGs) in such fiber were found to be sensitive to relative humidity, temperature and strain. This is in contrast to glass fiber FBGs which do not show any intrinsic humidity sensitivity [4]. The affinity for water of PMMA leads to a swelling of the fiber and an increase of refractive index, both of which contribute to an increase in the Bragg wavelength of a FBG written in the fiber [5]. This is a potentially very useful property, which has possible applications in chemical processing, agriculture, food storage, paper manufacturing, semiconductor and pharmaceutical industries, where humidity is monitored and controlled to ensure product quality. POFBGs have recently been applied to quantifying the small amount of water dissolved in aviation fuel [6].

Typical POFBG humidity sensor is made of poly(methyl methacrylate) (PMMA) based optical fiber. A change of environmental condition could lead to the water content change in the PMMA, which is a function of time [7]. This process of water absorption or desorption in PMMA can be described by the diffusion theory of mass transfer. Although the grating sensors made of different PMMA fibers may exhibit different response time, for the same type of PMMA fiber the response time of grating sensor is determined by the size of fiber diameter. In our previous work [8] the response time of POFBG was improved by reducing the fiber diameter using chemical etching. For some POFs which usually exhibit long response times a large part of fiber cladding need to be etched away so to achieve a satisfactory response time. This can lead to a fragile POFBG structure or even a failed guiding mode. A slotted PMMA optical fiber was suggested for further improvement in time response in [9]. However, the concerned fiber is not commercially available. The ultrashort laser pulses have been providing an effective microfabrication method to achieve spatial localized modification in materials inducing permanent changes on the surface or into the bulk of materials [10-11]. In the last few years, different laser microfabrication methods have been explored to lead to microstructures with enhanced optical, chemical and biological properties $[12,13]$.

*w.zhang@aston.ac.uk; phone: +44 (0) 121 2043549; fax: +44 (0) 121 2043682; http://www.aston.ac.uk/eas/

*x.chen@bangor.ac.uk; phone: +44 (0) 1248 382480; fax: +44 (0) 1248 361429; http://www.bangor.ac.uk/eng/ 
In this work we modified the POFBG by using laser etching technique. Excimer laser was utilized to etch the PMMA optical fiber and create the microstructures (slot, D-shape) in POFBGs. A significant improvement of response time of POFBG humidity sensing was achieved.

\section{PRINCIPLE \& FBG FABRICATON}

The PMMA based POF contained a $5 \mathrm{~mm}$ long FBG fabricated by illuminating from above a phase mask placed on top of the POF using 325nm UV light from a HeCd laser. Due to the high optical loss of POF in the $1550 \mathrm{~nm}$ region [14] a short length of POF was generally used to construct a POFBG sensor, which was then connected to a standard singlemode silica fiber down-lead. POF cannot be cleaved in the same fashion as silica fiber, neither can it be spliced using a fusion splicer. A UV gluing technique was developed [1] in which POF was cut by using hot knife to get a clean end face, then butt-coupled with a single-mode silica fiber angled pigtail; the butt-coupling joint was glued with a UV curable glue.

The Bragg wavelength of a PMMA based fiber grating depends on the effective core refractive index $n_{\text {eff }}$ and the grating pitch $\Lambda$, both of which are a function of temperature $T$ and the water content $w$. For constant temperature, the Bragg wavelength change of a POFBG against humidity can be expressed as [7]

$$
\Delta \lambda_{B}=\lambda_{B}(\eta+\beta) \Delta \mathrm{H}
$$

where $\lambda_{B}$ is the initial Bragg wavelength, $\eta$ is the normalized refractive index change with humidity $(\% \mathrm{RH})^{-1}$ and $\beta$ is the swelling coefficient related to humidity induced volumetric change $(\% \mathrm{RH})^{-1}$. If a POFBG is fabricated in a slotted fiber water or moisture could reach the fiber core in much less time, producing a fast response time. In our previous work different POFs were investigated by using chemical etching where some POF (e.g., POF1 in [8]) showed slow response time. Significant reduction of fiber diameter therefore is required to improve response time, which usually leads to unstable POFBG performance and fragile structure. In this work we intend to improve the POFBG performance made of this type of POF by laser etching technique.

\section{LASER ETCHING}

Excimer lasers can produce light in the deep ultraviolet region of the spectrum and most polymer materials will absorb such radiation. Hence excimer laser microstructuring has been used as an interesting approach to process polymeric materials $[10,11]$. Laser etching occurs when the laser beam interacts with a material and its molecular bonds are broken by the absorption of the UV photons. The response of the materials to laser pulse will depend on the optical absorption and the photochemical, thermal and mechanical effects.

(a)

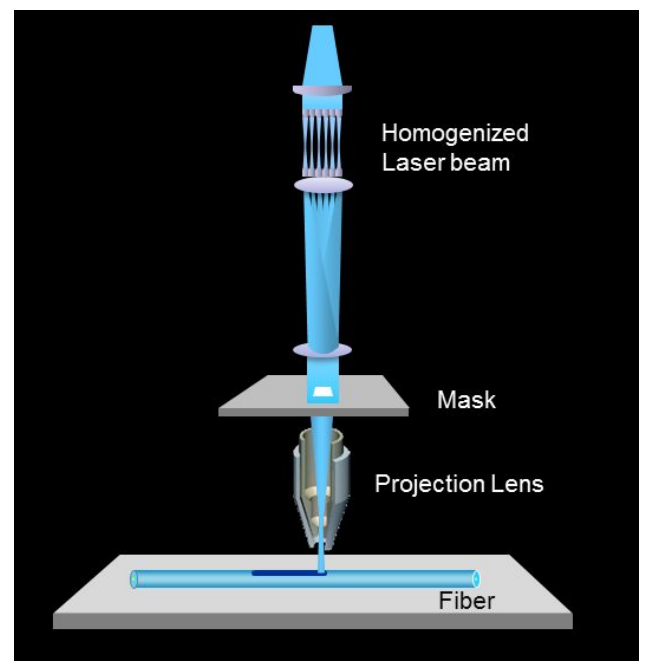

(b)

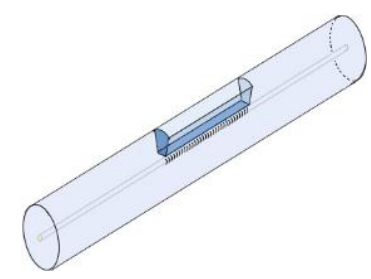

(c)

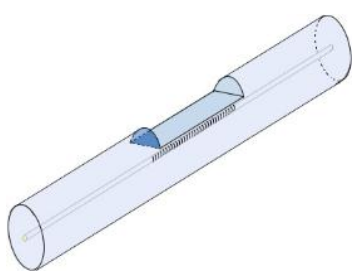

Fig. 1 Schematic diagram of (a) the excimer laser and mask projection scanning system, (b) slotted POFBG, and (c) D-shaped POFBG. 
To produce a designed microstructure on a sample, the excimer laser incorporating the mask projection method was used. Fig. 1(a) schematically illustrates the excimer laser micromachining system, which operated at $248 \mathrm{~nm}(\mathrm{KrF})$ laser beam and contained XY mask stage and a high precision air-bearing work piece handling stage. The laser beam was homogenized to produce a uniform intensity profile at the mask plane, then the mask image (square aperture) was projected through a high-resolution projection lens onto the surface of a PMMA fiber sample which was fixed on a micro-positioning work piece stage. Using a mask of $0.5 \mathrm{~mm} \times 0.5 \mathrm{~mm}$ in size and a projection lens with a demagnification of $\times 10$, a workpiece beam of $50 \mu \mathrm{m} \times 50 \mu \mathrm{m}$ in size and fluences of $1.37 \mathrm{~J} / \mathrm{cm}^{2}$ can be created. The microstructures were achieved by moving fiber sample under the static aperture laser beam and triggering the laser output firing with a defined incremental distance movement of work piece stage along the fiber axial direction. The slot was formed with the workpiece beam size smaller than fiber diameter while the D-shape was etched with beam size larger than fiber diameter.

Three POFBG samples (cladding diameter of $130 \mu \mathrm{m}$ ) were selected for laser etching. The first POFBG was created with a slot groove with depth of $33.1 \mu \mathrm{m}$, width of $100 \mu \mathrm{m}$ and longitudinal length of $5 \mathrm{~mm}$ where FBG underneath. Other two POFBGs were produced D-shaped structure with length of $5 \mathrm{~mm}$, but with different depth of $44.5 \mu \mathrm{m}$ and $50.5 \mu \mathrm{m}$, named as 'D-shaped 1' and 'D-shaped 2', respectively.

\section{EXPERIMENTAL RESULTS}

The POFBGs were placed inside an environmental chamber (Sanyo Gallenkamp) to allow operation in a controlled temperature and humidity environment. The wavelengths of the POFBGs were monitored by using an IBSEN I-MON 400 wavelength interrogation system. The environmental chamber was set at constant temperature $\left(24^{\circ} \mathrm{C}\right)$ and relative humidity (RH) varying from $40 \%$ to $90 \%$ with a step of $10 \% \mathrm{RH}$. Four POFBGs were put in test: one was normal POFBG without any modification located at $\sim 1535 \mathrm{~nm}$, one was slotted POFBG located at $1563 \mathrm{~nm}$, and other two were D-shaped POFBGs located at $1572 \mathrm{~nm}$. The wavelength responses of four POFBGs over humidity change were recorded by wavelength interrogation system. The captured responses are plotted in Fig. 2(a) where the traces for four POFBGs are wavelength shifted to give a better view. The step-changed relative humidity in environmental chamber was measured by using built-in sensor and is plotted in Fig. 2(a) as well.

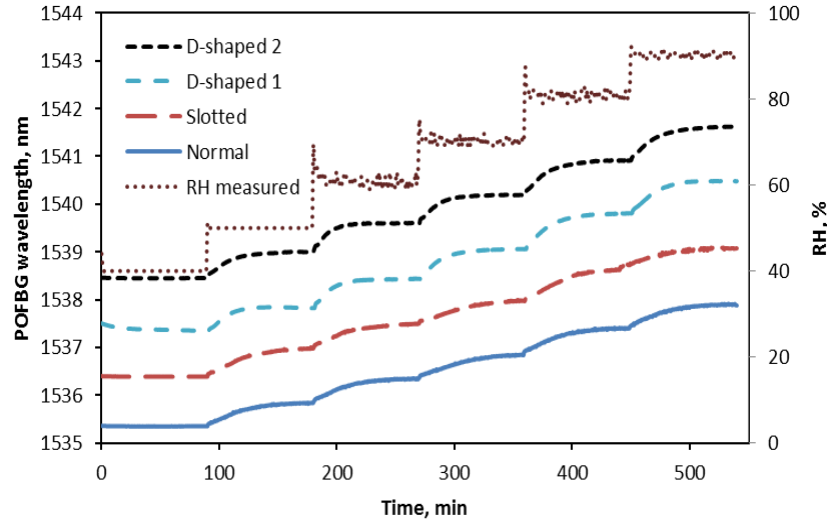

(a)

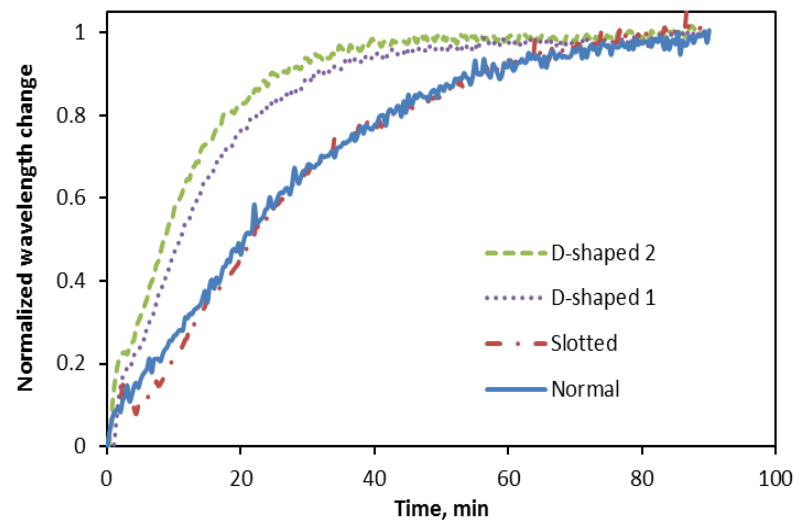

(b)

Fig. 2 (a) Wavelength responses of 4 POFBGs step-changed from $40 \%$ to $90 \% \mathrm{RH}$ at $24^{\circ} \mathrm{C}$, the traces for 4 POFBGs are wavelength shifted to give a better view; (b) Relative wavelength changes of POFBG sensors against one step RH change $(60 \%-70 \%)$.

To observe the response times of the different POFBG humidity sensors the measured humidity responses for the relative humidity step-changed from $60 \%$ to $70 \%$ are shown in Fig. 2(b). To simplify the comparison of the sensors which have different Bragg wavelengths, we chose to plot the time response of the relative wavelength change, $\Delta \lambda / \Delta \lambda_{\max }$, which is the ratio of the grating's Bragg wavelength deviation from its original value to the maximum wavelength deviation induced by the humidity change [8]. The response time then was estimated as the time of relative wavelength change being increased to $90 \%$.

For the normal POFBG without any laser etching the response time was estimated as $54 \mathrm{~min}$, which was in agreement with 53 min reported in our previous work [8]. For the slotted POFBG the response almost overlapped with that of 
normal POFBG, so was the response time. The D-shaped POFBGs showed a much improved response time, estimated as 30min and 24 min for 'D-shaped 1' and 'D-shaped 2', respectively.

From Eq. (1) one can see that the wavelength change of POFBG induced by environmental humidity consists of two parts: humidity dependent refractive index change and humidity induced volumetric change in fiber core. If the moisture can reach the fiber core in short time it means a fast response time. The slot etched by using excimer laser has a depth of $\sim 33.1 \mu \mathrm{m}$. It does not reach the fiber core but allows the moisture into the fiber core more quickly. From the wavelength response of slotted POFBG in Fig. 2(b), it shows a quick change in the start then it drops a little bit; eventually it overlaps with that of normal POFBG, indicating that the moisture does reach the fiber core more quickly hence to produce a quick change of Bragg wavelength in the initial stage. However, the volumetric change in fiber core not only depends on the moisture reaching the core but also on the volumetric change in fiber cladding. Since the volume of fiber cladding is much larger than that of fiber core, the humidity induced volumetric change is dominated by fiber cladding. From the slotted POFBG response one can see that there is a lag after the quick start. This is a lag between the humidity induced refractive index change in fiber core and the volumetric change contribution from fiber cladding. Since the slot only takes a small part of the volume of the POFBG cladding the response time of slotted POFBG is very close to that of normal POFBG. This can also explain the response of D-shaped POFBG. There exist a quick start and a lag in the Dshaped POFBG response. This lag is caused by the delayed volumetric change contribution from fiber cladding. Since the laser-etched D-shape takes off a significant part of the POFBG cladding, the volumetric change of the cladding takes less time to complete. Consequently, the lag is smaller than that of slotted POFBG response, and overall response time is much improved. The response time of the deeply etched POFBG ('D-shaped 2') exhibits the fastest response time of 24 min, showing great improvement over the normal POFBG.

\section{CONCLUSION}

The response time of POFBG over humidity is influenced by many factors like the composition of dopant and copolymer used, drawing process, size etc. For the same type of POF the size of the fiber diameter plays a major role. Laser etching can provide a flexible method to modify POFBG sensor performance. In this work we have created the microstructures (slot, D-shape) over POFBG. The latter significantly improves the humidity response performance of POFBG sensor. By the use of laser etching technique, the slot and D-shape with different depth can be micromachined to define different response time to meet the requirements of different applications. For those POFs, which normally exhibit large response time, this technique can enhance POFBG sensor performance without weakening the sensor structure and signal transmission. Also the technique can create more designated structures in both polymer and silica optical fibers/fiber gratings to extend the applications in sensing and communication systems.

\section{ACKNOWLEDGMENT}

The authors would like to acknowledge the support from the projects of FP7/2007-2013 No. 314032, FP7 PIRSES-2013612267, and the Sêr Cymru NRN.

\section{REFERENCES}

[1] D. J. Webb, and K. Kalli, Fiber Bragg Grating Sensors, A. Cusano, A. Cutolo and J. Albert ed., Bentham Science Publishers Ltd, 2011.

[2] Z. Xiong, G. D. Peng, B. Wu, and P. L Chu, IEEE Photon. Technol. Lett., Vol. 11, 352-354, 1999.

[3] H. Dobb, D. J. Webb, K. Kalli, A. Argyros, M. C. J. Large, and M. A. van Eijkelenborg, Opt. Lett., Vol. 30, 3296-3298, 2005

[4] C. Zhang, W. Zhang, D. J. Webb, and G.-D. Peng, Electron. Lett., Vol. 46, 643-644, 2010.

[5] N. G. Harbach, Ph.D. dissertation, Inst. Appl. Opt., EPFL, Lausanne, Switzerland, 2008.

[6] C. Zhang, X. Chen, D. J. Webb, and G.-D. Peng, Proc. of SPIE 7503, 750380-1, 2009.

[7] W. Zhang, D. J. Webb, Opt. Lett., Vol. 39 (10), 3026-3029, 2014

[8] W. Zhang, D. J. Webb, and G.-D. Peng, J. Lightw. Technol., Vol. 30 (8), 1090-1096, 2012.

[9] F. M. Cox, M. C. J. Large, C. M. B. Cordeiro, R. Lwin, A. Argyros, Proc. of SPIE 7004, 700427-1, 2007.

[10] P. E. Dyer, Appl. Phys. A, Vol. 77, 167-173, 2003.

[11] A. S. Holmes, Science, Measurement and Technology, IEE Proceedings, Vol. 151 (2), IET, 2004.

[12] J. Burt, A. Goater, A. Menachery, R. Pethig, and N. Rizvi, J. Micromech. Microeng., Vol. 17, 250-257, 2007.

[13] G. N. Smith, T. Allsop, K. Kalli, C. Koutsides, R. Neal, K. Sugden, P. Culverhouse, and I. Bennion, Opt. Express, Vol. 19 (1), $363-370,2011$.

[14] A. Stefani, W. Yuan, C. Markos, and O. Bang, IEEE Photon. Technol. Lett., Vol. 23 (10), 660-662, 2011 